Received: 23 May 2017

Accepted: 18 September 2017

Published online: 29 September 2017

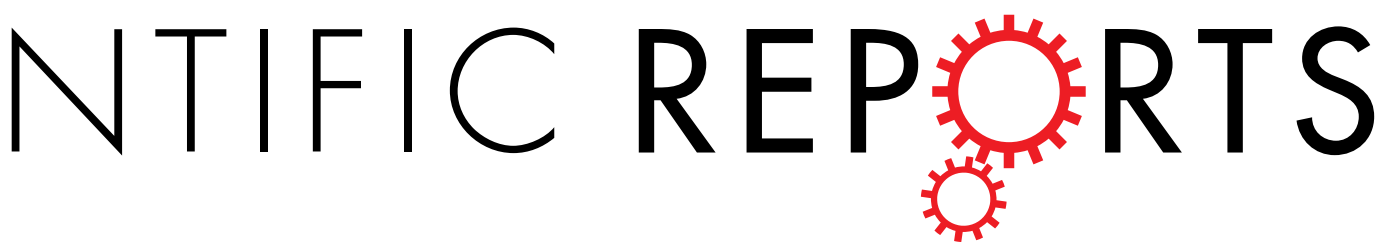

\title{
OPEN The Equivalence Between Unit-Cell Twinning and Tiling in Icosahedral Quasicrystals
}

\author{
Albert Prodan, Ram Dušić Hren, Marion A. van Midden, Herman J. P. van Midden \& \\ Erik Zupanič
}

It is shown that tiling in icosahedral quasicrystals can also be properly described by cyclic twinning at the unit cell level. The twinning operation is applied on the primitive prolate golden rhombohedra, which can be considered a result of a distorted face-centered cubic parent structure. The shape of the rhombohedra is determined by an exact space filling, resembling the forbidden five-fold rotational symmetry. Stacking of clusters, formed around multiply twinned rhombic hexecontahedra, keeps the rhombohedra of adjacent clusters in discrete relationships. Thus periodicities, interrelated as members of a Fibonacci series, are formed. The intergrown twins form no obvious twin boundaries and fill the space in combination with the oblate golden rhombohedra, formed between clusters in contact. Simulated diffraction patterns of the multiply twinned rhombohedra and the Fourier transform of an extended model structure are in full accord with the experimental diffraction patterns and can be indexed by means of three-dimensional crystallography. The alternative approach is fully compatible to the rather complicated descriptions in a hyper-space.

Ever since quasicrystals (QCs) were first reported ${ }^{1}$ they attracted great interest, because they apparently contradicted some basic concepts of crystallography ${ }^{2-4}$. Contrary to fully disordered solids and perfectly grown single crystals, characterized by their rotational and translational symmetries, QCs with their forbidden five-fold rotational symmetry and with the apparently lost translational order represented something in between the two categories. However, some of their properties contradict this distinction. Their shapes can be well developed and the corresponding diffraction patterns (DPs) show exceptionally sharp reflections, without any diffuse scattering, characteristic of short-range order, modulation, or any other deviation from an ideal crystalline structure.

Pauling was convinced that none of the existing crystallographic rules was violated in the newly discovered materials ${ }^{5-10}$. He believed these crystals were composed of twinned cubic domains with huge unit cells, whose basic building elements were composed of one $\mathrm{Mn}$ atom linked to twelve $\mathrm{Al}$ atoms. Although the existing experiments seemingly supported his model, he after all run into problems. Another major problem with Pauling's approach was, that no twin boundaries were ever detected in QCs ${ }^{11}$.

Contrary to Pauling, a number of researchers ${ }^{12-18}$ considered QCs an exception to the known solid state structures, which required a novel approach. Their explanation was based on the so-called Amman tiling ${ }^{19}$, the three-dimensional equivalent of the two-dimensional Penrose tiling. Likewise to two Penrose rhombic tiles filling a plane, their three-dimensional equivalents, the prolate and the oblate golden rhombohedra, will fill the space and form the QC structure.

It is shown in the present work that tiling in the icosahedral QC structure can also be properly explained by cyclic unit cell twinning ${ }^{20,21}$, applied on primitive golden rhombohedra, forming thus intergrown twins without explicit twin-boundaries.

\section{Results}

Procedure. Instead of describing a QC structure in a hyper-space, the present description is based on twinning of the basic building units.

Dependent on the sizes and the composition of the constituent atoms a hypothetical parent face-centered cubic structure of an alloy may collapse into a primitive rhombohedral one along four equivalent directions. If the resulting rhombohedral angle is close to $63.43^{\circ}$, i.e. the angle of a prolate golden rhombohedron, it will lock 
into that angle and fill the local space by a five-fold cyclic twinning. Clusters with five-fold rotational symmetry will be formed, with the rhombohedra of adjacent clusters in contact stacked either in-phase, or will leave oblate interstitials.

The procedure of reconstructing the corresponding DPs was performed in two steps. First, the DPs of the individual golden rhombohedra of both types and their clusters were considered, giving information on the rotational symmetry of the constituent clusters. Second, Fourier transforms of larger structural models, composed of the clusters in contact were calculated and compared with the experimental DPs. The Fourier transforms should give information on both, the rotational as well as all possible translational periodicities, present in the model QC structure.

The structural units and their stacking. First, the structure of the icosahedral star polyhedra and the corresponding DPs were considered. Twinned prolate golden rhombohedra were combined into a rhombic hexecontahedron (RH $)^{22}$ with the corresponding DPs constructed by overlapping individual contributions. No contributions from additional long-range ordering, formed across clusters surrounding the RHs, were considered at this stage.

The prolate $\left(a=0.435 \mathrm{~nm}, \alpha_{p}=63.43^{\circ}\right)$ and the oblate $\left(a=0.435 \mathrm{~nm}, \alpha_{o}=116.57^{\circ}\right)$ golden rhombohedra with $\alpha_{p}+\alpha_{o}=180^{\circ 23,24}$ were obtained by deforming a parent face-centered cubic structure with a random occupation of both constituent atoms, in accord with the stoichiometry of the compound. The unit cell edge was chosen to fit the experimental DPs of $\mathrm{MnAl}_{6}$, a representative of the icosahedral QCs. The rhombohedral angle was determined to fit the five-fold cyclic twinning with a rotational angle of $(360 / 5)^{\circ}=72^{\circ}$ around the rhombohedral $\langle 100\rangle$ axes and with the twin-planes corresponding to the rhombohedral $\{100\}$ planes. The diagonals along the three-fold axes make $d_{p}=1.037 \mathrm{~nm}$ for the prolate and $d_{o}=0.245 \mathrm{~nm}$ for the oblate golden rhombohedra. In case of $\mathrm{MnAl}_{6}$ and for the given parameters both constituent atoms are obviously too large (the atomic radii of $\mathrm{Mn}$ and $\mathrm{Al}$ are $0.135 \mathrm{~nm}$ and $0.143 \mathrm{~nm}$, respectively) to be accommodated along the short diagonals of the oblate units. Consequently, the positions connecting the diagonals of the oblate rhombohedra can be only partly occupied. The shapes of both types of golden rhombohedra are determined by the shapes of their rhombic faces, whose diagonals are determined by the golden ratio $\varphi=(1+\sqrt{5}) / 2 \approx 1.61803$. Twenty prolate units are needed to construct a RH, whose origin represents the center of the cluster formed around it. The remaining empty spaces between adjacent clusters of various sizes, which cannot be filled by the prolate rhombohedra, represent the oblate rhombohedral interstices of altogether thirty possible orientations.

The DPs of the twinned prolate and oblate rhombohedra were constructed under two assumptions. First, in accord with the experimental DPs, where the first-order low-index reflections appear much stronger in comparison with the weak second-order ones, only reflections with Miller indices $\overline{111} \leq h k l \leq 111$ were included. Second-order reflections may be observed in over-exposed diffraction patterns, but if present at all, they will appear very weak. Second, to exclude reflections from higher Laue zones the lengths of the "spikes" were kept below $\Delta S \leq 0.18 \mathrm{~nm}^{-1}$. Any other values in accord with the requirement to show all reflections belonging to the zero-order Laue zone and to exclude contributions from higher-order zones will give the same result. All rhombohedra, belonging to the same RH, were interrelated by rotating the starting rhombohedron into all possible twinned positions.

Similar twinned constructions were composed with the oblate golden rhombohedra, representing the interstices between the prolate units of the clusters in contact. Contributions from both polyhedral types to the DPs along the same directions were overlapped and positions of possible dynamical scattering were determined. This was done to show that any dynamical scattering will coincide with the reflections belonging to the Fibonacci series, discussed further down. As an example, the resulting overlapped DP along one of the twelve equivalent five-fold $\langle 01 \phi\rangle$ (i.e. approximately $\langle 0,34,55\rangle$ ) QC zone-axes is shown in Fig. 1.

To simulate the DPs along any of the $\langle 111\rangle$ zone axes, all twenty twinned prolate rhombohedra, belonging to a RH were rotated by $37.38^{\circ}$, i.e. the angle between the rhombohedral $\langle 100\rangle$ and $\langle 111\rangle$ directions, which correspond to the $\langle 01 \phi\rangle$ five-fold and the $\langle 111\rangle$ three-fold directions of the RH. By following the same procedure as in case of the five-fold zone axes, DPs along the three-fold axes, or along any other direction can be completed. The calculated DPs along the $\langle 01 \phi\rangle$ and $\langle 111\rangle$ icosahedral zone axes fit very well with the published experimental DPs of icosahedral QCs ${ }^{9,25-27}$. Since none of the published experimental DPs was indexed, comparisons with less symmetric zones were not undertaken.

The translational symmetry and the Fourier transform of an extended structural model. Next, the ordering between adjacent clusters, formed around particular RHs, was considered. The apparently lost translational symmetry in QCs was the main reason for classifying them as exceptions with regard to normal single crystals. The distances between multiply twinned five-fold rotational centers vary throughout the QC structure and form clusters of variable sizes around individual RH centers. It was recently shown how the icosahedral QCs grow, how this growth is influenced by defects and how a wrong initial stacking is modified during further growth $^{28}$. Regardless of the actual growing mechanism and the corresponding accommodation of the units composing the structure, the prolate as well as the oblate rhombohedra in contact across adjacent clusters are kept in exact phase relationships. As a result series of distinct periodicities are formed along equivalent directions, interrelated as members of a Fibonacci series. The actual periodicities depend on the orientations of the polyhedra, joining the clusters. The entire crystalline space is thus composed of intergrown twins without any obvious boundaries. The long-range translational order along all equivalent directions depends on the actual sequences of the differently oriented prolate and oblate rhombohedra. Consequently, the reflections in the reciprocal space, belonging to the series of periodicities in the crystal, are sharp and comparable to those of perfectly ordered single crystals. Thus, the apparently lost long-range order, being a result of the five-fold twinning, is replaced by a 


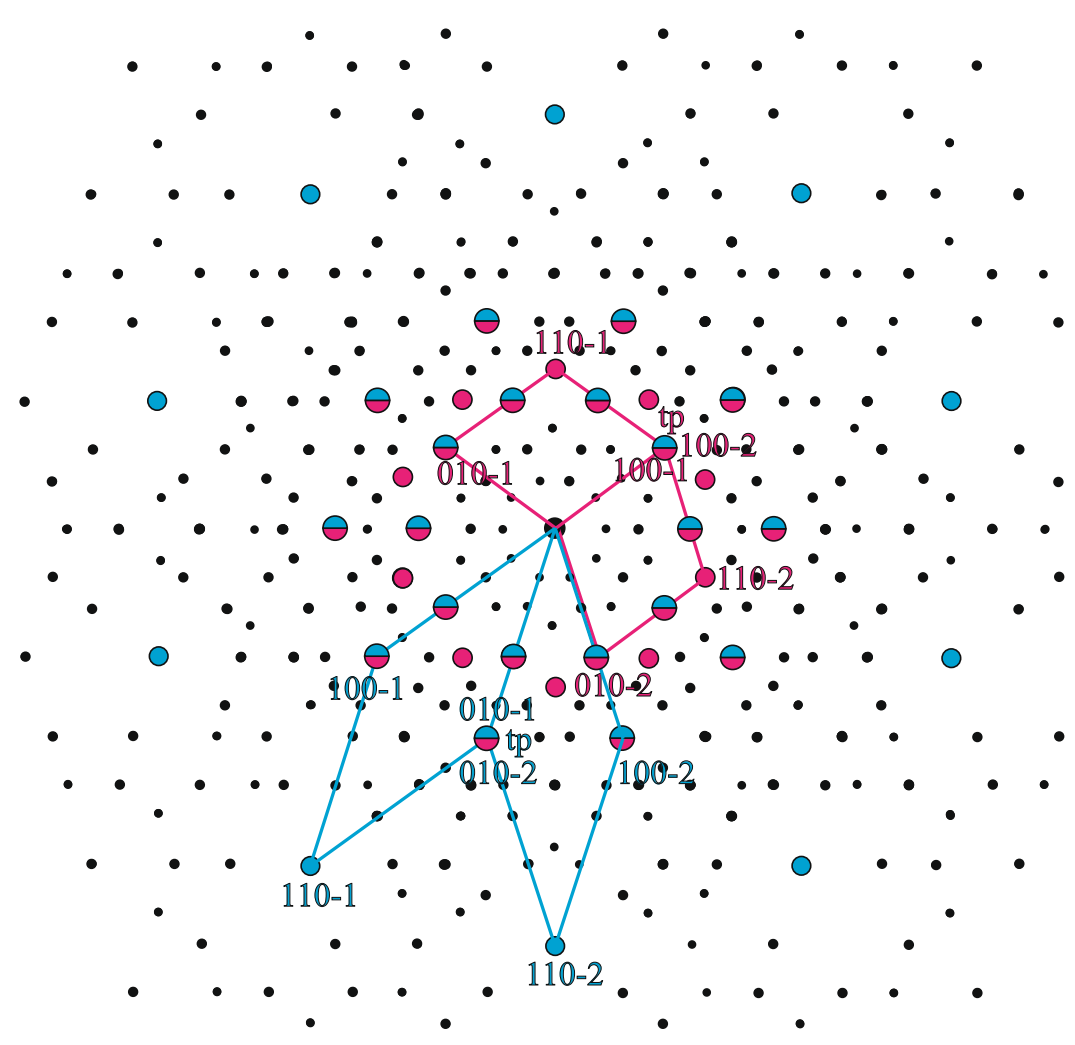

Figure 1. The simulated diffraction pattern along one of the rhombic hexecontahedron five-fold axes, obtained by overlapping contributions from the prolate and the oblate twinned units. Two of five prolate (red) and oblate (blue) unit cells are indexed and their twinning plane (tp) indicated. Positions, where contribution from possible dynamical scattering might be found, are indicated by small black dots.

series of long-periodicities, all of them in proper phase-relationships, like the structure was continuous and not intergrown.

To study the contribution of the specific long-range ordering in the icosahedral QCs, a larger model structure, composed of twinned golden rhombohedra of both types, was constructed of altogether 1603 atoms of a single kind. These atoms represent e.g. in case of $\mathrm{MnAl}_{6}$ the disordered constituent $\mathrm{Mn}$ and $\mathrm{Al}$ atoms in their proper stoichiometric ratio. The structure is shown in Fig. 2, clearly showing the RH centers and the oblate rhombohedra interconnecting the clusters.

Sections through the three-dimensional Fourier transform (FT) of this model structure, performed perpendicular to one of the twelve equivalent five-fold axes is shown in Fig. 3. These sections correspond to the experimental DPs along the same zone axis.

\section{Discussion}

Five-fold twinning is a known crystal growing mechanism, most often found in nanoparticles and thin films $\mathrm{s}^{30}$. However, the twinning operation in these cases is applied to a crystal as a whole and thus limited to a single five-fold rotation. The icosahedral QCs with the specific structure can be likewise considered a result of cyclic twinning at the unit-cell level ${ }^{20,21}$, i.e. with the twinning operation applied on the primitive golden rhombohedra. If a parent face-centered cubic cell is deformed into either of the four equivalent primitive prolate golden rhombohedra with $\alpha_{p}=63.43^{\circ}$, the space can be locally filled by forming RHs with twenty twinned prolate rhombohedra each. Clusters, formed around these RH star polyhedra, cannot be stacked together without leaving interstices in the form of oblate golden rhombohedra. However, the long-range order in icosahedral QCs is fully preserved. Instead of a single periodicity, a series of periodicities, interrelated as members of a Fibonacci series, is formed along equivalent directions. These periodicities depend on the orientation and the sequence of the prolate rhombohedra and the oblate interstices, which fill the remaining space. Consequently, QCs are twinned single crystals with preserved rotational, but in a certain way also translational symmetry. Since the twins are intergrown, they do not show clear twin-boundaries. The related long-range periodicities are determined by combinations of long and short segments, usually considered as sections through a higher-dimensional space ${ }^{2}$. Intergrowth in QCs was suggested before already, e.g. by Mackay ${ }^{31}$ and in one of the papers ${ }^{32}$, where the QC structure was considered an interpenetrating incommensurately modulated structure, described as a three-dimensional section through a higher-dimensional space ${ }^{32-35}$. The related periodicities were described as structural modulations, induced by interacting intergrown subsystems $\mathrm{s}^{32}$. However, though mathematically elegant, descriptions in higher dimensions are connected with difficulties, lacking i.a. exact information on structural parameters, composition and consequently structure-to-properties relationships. A description by means of cyclic unit-cell twinning is fully 

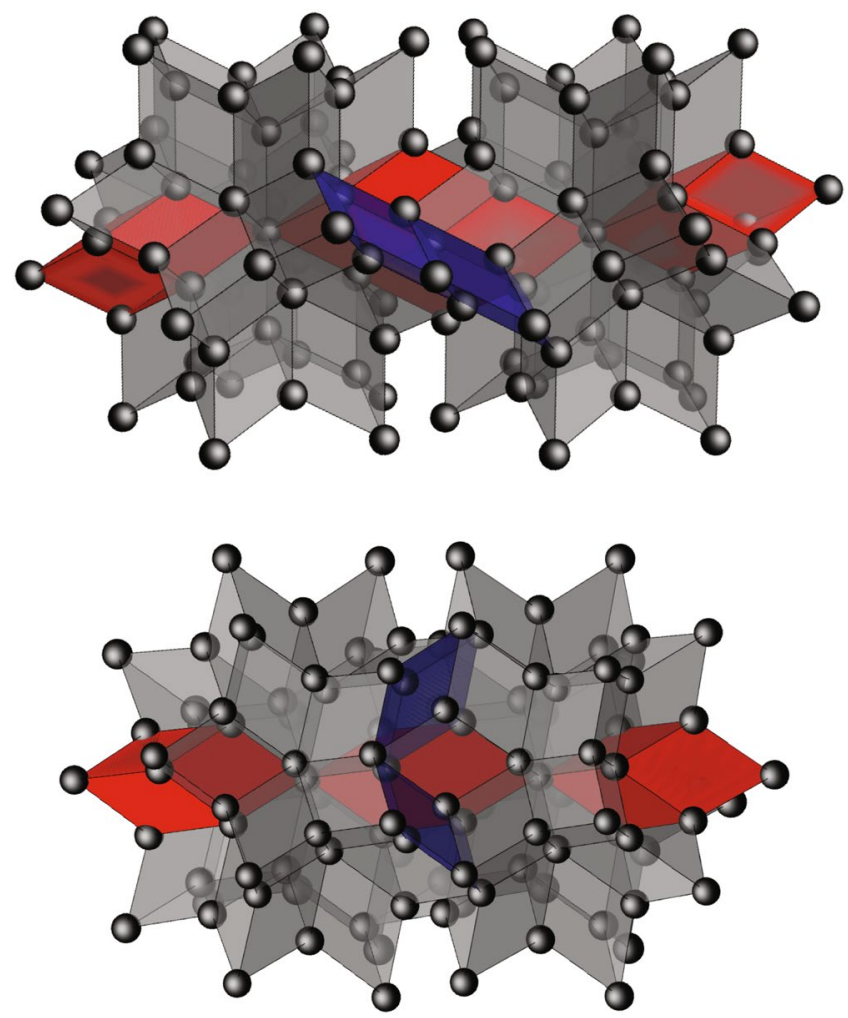

Figure 2. Two possible stackings of adjacent rhombic hexecontahedra. Two of the twenty prolate rhombohedra in both star polyhedra, interrelated by a center of symmetry, are drawn red to point out their stacking. Two oblate rhombohedra are drawn blue.

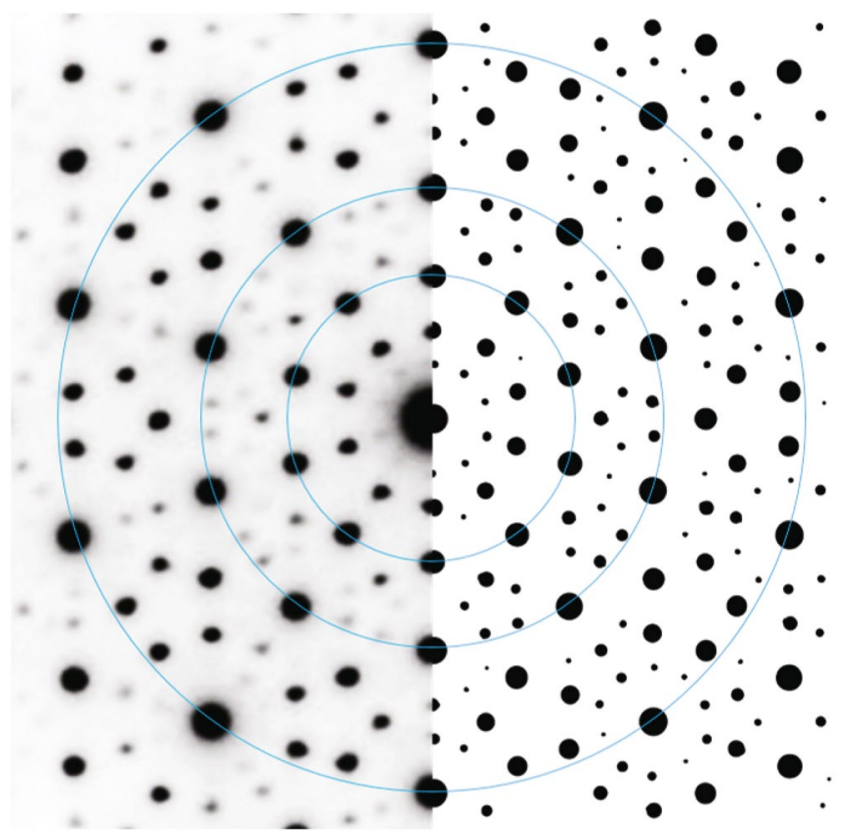

Figure 3. A section through the Fourier transform of the model icosahedral QC structure (right) and the corresponding electron diffraction pattern, recorded along one of the twelve equivalent $\langle 01 \phi\rangle$ icosahedral zone axes $(\text { left })^{29}$. 
equivalent with all other approaches and the structure can be considered by means of three-dimensional crystallography with all its advantages. There is also no need for considering the QC structure as being incommensurately modulated. Numerous cases are known, e.g. many quasi one-dimensional modulated structures ${ }^{36}$, where descriptions in higher dimensions were replaced by alternative notations, describing separately the basic and the modulated structures. The icosahedral QCs represents yet another case, where the incommensurate modulation can be replaced by a series of translational periodicities.

Pauling's approach to the problem was obviously not adequate. Regardless of all arguments brought up in the debates that followed the discovery of QCs, he run into problems, because he tried to prove his ideas by applying twinning onto huge icosahedral unit cells. There are no forces in nature, able to act between atoms at distances of the order of nanometers and larger. Structures with huge unit cells can only be formed as a result of two or more competing mechanisms, whose relatively short periodicities coincide at large distances. That is also what makes the QC structure a specific case.

Multiple unit-cell twinning must be energetically favorable in comparison with the untwinned rhombohedral structure. Whether a parent structure will collapse into a less symmetric one will in metallic alloys depend on the size of the constituent atoms and the stoichiometry of the compound. Twinning of golden rhombohedra is thus a result of the need to reduce the energy and to fill the space accordingly.

It is well known that the DPs of high-quality QCs show exceptionally sharp reflections, whose full widths at half maxima of less than $0.001^{\circ}$ are of the order of the $\mathrm{x}$-ray instrumental resolution ${ }^{37,38}$. Both, the sharp reflections and the very clear background are proofs of a near perfect ordering over large distances, comparable to the best ordered metallic crystals. This is supported by the fact that any multiple scattering coincides with the reflections belonging to distinct long-range periodicities of the Fibonacci series.

\section{Conclusions}

It is shown that tiling in icosahedral QCs is fully equivalent to cyclic twinning at the unit-cell level, with the twinning operation applied on primitive prolate golden rhombohedra. Multiple twinning of these rhombohedra forms centers with five-fold rotational symmetry and results in distinct long-range periodicities, interrelated as members of a Fibonacci series. The space is locally filled with RH stars of twenty prolate golden rhombohedra, while the interstices between adjacent clusters, formed around the RH centers, represent oblate golden rhombohedra of thirty possible orientations.

The intergrown twinned QC structure shows no explicit twin-boundaries. It can be properly described in the real three-dimensional space, with all its advantages in comparison with a description in a hyper-space.

\section{References}

1. Shechtman, D., Blech, I., Gratias, D. \& Cahn, J. W. Metallic phase with long-range orientational order and no translational symmetry. Phys. Rev. Lett. 53, 1951-1953 (1984).

2. Gratias, D. The adventure of quasicrystals: a sucessful multidisciplinary effort. Europhysics News 43, 26-29 (2012).

3. The Oregon State University Libraries Special Collections \& Archives Research Center. The Pauling Blog, Part 1-4. https:// paulingblog.wordpress.com/tag/quasicrystals (2012).

4. Kramer, P. Gateways towards quasicrystals 1101.0061v1[cond-mat.mtrl-sci] (2010)

5. Shechtman, D. \& Blech, I. A. The microstructure of rapidly solidified Al6Mn. Metallurgical Transactions A 16, 1005-1012 (1985).

6. Stephens, P. W. \& Goldman, A. I. Sharp diffraction maxima from an icosahedral glass. Phys. Rev. Lett. 56, 1168-1171 (1986).

7. Pauling, L. Apparent icosahedral symmetry is due to directed multiple twinning of cubic crystals. Nature 317, 512-514 (1985).

8. Pauling, L. The nonsense about quasicrystals. Science News 129 (1986).

9. Pauling, L. So-called icosahedral and decagonal quasicrystals are twins of an 820-atom cubic crystal. Phys. Rev. Lett. 58, 365-368 (1987).

10. Pauling, L. Icosahedral quasicrystals of intermetallic compounds are icosahedral twins of cubic crystals of three kinds, consisting of large (about 5000 atoms) icosahedral complexes in either a cubic body-centered or a cubic face-centered arrangement or smaller (about 1350 atoms) icosahedral complexes in the beta-tungsten arrangement. Proc. Nat. Acad. Sci. USA 86, 8595-8599 (1989).

11. Steinhardt, P. \& Ostlund, S. (eds.) The Physics of Quasicrystals 310-12. (World Scientific Publishing, 1987).

12. Cahn, J., Gratias, D. \& Shechtman, D. Pauling's model not universally accepted. Nature 319, 102 (1986).

13. Mackay, A. L. Pauling's model not universally accepted. Nature 319, 103 (1986).

14. Bancel, P. A., Heiney, P. A., Stephens, P. W. \& Goldman, A. I. Pauling's model not universally accepted. Nature 319, 103 (1986)

15. Berezin, A. A. Pauling's model not universally accepted. Nature 319, 104 (1986).

16. Heiney, P. A., Bancel, P. A. \& Horn, P. M. Comment on "So-called icosahedral and decagonal quasicrystals are twins of an 820 -atom cubic crystal". Phys. Rev. Lett. 59, 2119-2119 (1987).

17. Bancel, P. A., Heiney, P. A., Horn, P. M. \& Steinhardt, P. J. Comment on a paper by Linus Pauling. Proc. Nat. Acad. Sci. USA 86(22), 8600-8601 (1989).

18. Lidin, S., Andersson, S., Bovin, J.-O., Malm, J. O. \& Terasaki, O. A structural model for a quasicrystalline material. Acta Crystallographica Section A 45, fc33-fc36 (1989).

19. Lord, E. A., Ranganathan, S. \& Kulkarni, U. D. Tilings, coverings, clusters \& quasicrystals. Current Science 78, 64-72 (2000).

20. Andersson, S. \& Hyde, B. Twinning on the unit cell level as a structure-building operation in the solid state. Journal of Solid State Chemistry 9, 92-101 (1974).

21. Hyde, B. G. \& Andersson, S. Inorganic Crystal Structures (John Wiley \& Sons, N.Y., 1989).

22. Guyot, P. News on file-fold symmetry. Nature 640-641 (1987).

23. Weber, S. Quasicrystals. http://www.jcrystal.com/steffenweber/qc.html (2015).

24. Knott, R. The Golden Geometry of Solids or Phi in 3 dimensions. http://www.maths.surrey.ac.uk/hosted-sites/R.Knott/Fibonacci/ phi3DGeom.html (2009).

25. Bindi, L., Steinhardt, P. J., Yao, N. \& Lu, P. J. Natural quasicrystals. Science 324, 1306-1309 (2009).

26. Guo, J. Q., Abe, E. \& Tsai, A. P. Stable icosahedral quasicrystals in binary Cd-Ca and Cd-Yb systems. Phys. Rev. B 62, R14605-R14608 (2000).

27. Wikipedia, The Free Encyclopedia. Quasicrystal. http://en.wikipedia.org/wiki/Quasicrystal (2017).

28. Hann, C. T., Socolar, J. E. S. \& Steinhardt, P. J. Local growth of icosahedral quasicrystalline tilings. Phys. Rev. B 94, 014113 (2016).

29. Materialscientist. Electron diffraction pattern of an icosahedral Zn-Mg-Ho quasicrystal, CC BY-NC-SA 2.0. https://commons. wikimedia.org/wiki/File:Zn-Mg-HoDiffraction.JPG (2010).

30. Hofmeister, H. Fivefold twinned nanoparticles (American Scientific Publishers, Stevenson Ranch, 2004). 
31. Mackay, A. L. What has the penrose tiling to do with the icosahedral phases? Geometrical aspects of the icosahedral quasicrystal problem. Journal of Microscopy 146, 233-243 (1987).

32. Spal, R. D. Interpenetrating incommensurately modulated lattices with icosahedral symmetry. Phys. Rev. Lett. 56, 1823-1826 (1986).

33. Cahn, J. W. \& Gratias, D. A structural determination of the Al-Mn icosahedral phase. J. Phys. Colloques 47, C3-415-C3-424 (1986).

34. Cahn, J. W., Gratias, D. \& Mozer, B. A 6-d structural model for the icosahedral (Al, Si)-Mn quasicrystal. J. Phys. France 49, $1225-1233(1988)$.

35. Duneau, M. \& Oguey, C. Ideal AlMnSi quasicrystal: a structural model with icosahedral clusters. J. Phys. France 50, 135-146 (1989).

36. Monceau, P. Electronic crystals: an experimental overview. Advances in Physics 61, 325-581 (2012).

37. Abe, E., Yanfa, Y. \& J. Pennycook, S. Quasicrystals as cluster aggregates. Nature Materials 759-767 (2004).

38. Kycia, S. W. et al. Dynamical x-ray diffraction from an icosahedral quasicrystal. Phys. Rev. B 48, 3544-3547 (1993).

\section{Acknowledgements}

We are thankful to dr. G. Dražić for supplying valuable information on electron diffraction from quasicrystals and to the Slovenian Research Agency (ARRS) for its financial support under Program No. P1-0099.

\section{Author Contributions}

A.P. and E.Z. conceived the experiment, A.P., R.D.H., M.A.M., H.J.P.M. and E.Z. conducted the experiments, A.P. and E.Z. wrote the manuscript. All authors analyzed and discussed the results and reviewed the manuscript.

\section{Additional Information}

Competing Interests: The authors declare that they have no competing interests.

Publisher's note: Springer Nature remains neutral with regard to jurisdictional claims in published maps and institutional affiliations.

(c) (i) Open Access This article is licensed under a Creative Commons Attribution 4.0 International License, which permits use, sharing, adaptation, distribution and reproduction in any medium or format, as long as you give appropriate credit to the original author(s) and the source, provide a link to the Creative Commons license, and indicate if changes were made. The images or other third party material in this article are included in the article's Creative Commons license, unless indicated otherwise in a credit line to the material. If material is not included in the article's Creative Commons license and your intended use is not permitted by statutory regulation or exceeds the permitted use, you will need to obtain permission directly from the copyright holder. To view a copy of this license, visit http://creativecommons.org/licenses/by/4.0/.

(C) The Author(s) 2017 